\title{
REFERENCE
}

1. Guidelines for the diagnosis and management of Barrett's columnar lined oesophagus. A report of the working party of the British Society of Gastroenterology. http://www.bsg.org.uk (accessed 17 October 2010).

\section{OC-071 DOES BARRETT'S SURVEILLANCE REALLY DETECT MORE DYSPLASIA?}

doi:10.1136/gut.2011.239301.71

S Zeki, S Oke 1, J Deacon, D Morris Gastroenterology, Queen Elizabeth II Hospital, Welwyn Garden City, UK

Introduction Barrett's oesophagus is a premalignant condition affecting $2 \%$ of the UK population with a lifetime risk of developing oesophageal adenocarcinoma of 1\%. UK Guidelines released in 2005 recommend endoscopic surveillance 2 yearly or sooner depending on histology. ${ }^{1}$ The impact of surveillance is contentious.

In our Trust formal Barrett's surveillance did not commence until after the guidelines were introduced - prior to this, the surveillance interval varied between the consultants. The introduction of the guidelines locally provided a unique opportunity for comparison with non surveillance.

Methods The hospitals involved serve a catchment area of 500,000 patients. Patient records were searched for all patients who underwent a gastroscopy for any indication and who had an endoscopic diagnosis of CLO between January 2004 and December 2009 at a district general hospital in the UK. Oesophageal dysplasia and cancer records were obtained separately and cross referenced with the CLO database.

\section{Results}

The results of 1270 gastroscopies detecting CLO in 791 patients are shown in table 1.

Conclusion Although the study was observational and not powered to show significant differences, the results suggest that in our population, CLO associated dysplasia is just as likely to be diagnosed in those undergoing surveillance as in symptomatic patients. This argues in favour of a surveillance programme as without it the number of detected dysplasias would be significantly less. Although not significant, more cancers were diagnosed on interim endoscopies in those already on a surveillance programme. This may argue for increased vigilance at the time of surveillance or a decreased interval between surveillance endoscopies. Randomised prospective studies such as the BOSS study will answer the question fully in time.

Competing interests None.

Keywords Barrett's adenocarcinoma, Barrett's oesophagus, surveillance.

Table 1 OC-071 Detection of dysplasia/ cancer in all patients with CLO at gastroscopy.

\begin{tabular}{|c|c|c|c|}
\hline & Surveillance gastroscopy & Symptom based gastroscopy & $\begin{array}{l}\text { p-value ( } 2 \text { tailed } \chi^{2} \text { with } \\
\text { Yates' correction) }\end{array}$ \\
\hline Total Number of Patients & 332 (41.9\%) & 459 (58.1\%) & \\
\hline Number of Individuals With Dysplasia & $16(4.8 \%)$ & $17(3.7 \%)$ & 0.57 \\
\hline Cancer & $1(0.3 \%)$ & $\begin{array}{l}4(0.9 \%) \text { - All patients already on } \\
\text { surveillance programme }\end{array}$ & 0.59 \\
\hline
\end{tabular}

